\title{
On the Origin of the $6.4 \mathrm{keV}$ line from the GRXE
}

\author{
Romanus Eze ${ }^{1,2}$, Kei Saitou ${ }^{1,3}$ and Ken Ebisawa ${ }^{1,3}$ \\ ${ }^{1}$ Japan Aerospace Exploration Agency, Institute of Space and Astronautical Science, \\ 3-1-1 Yoshinodai, Chuo-ku, Sagamihara, Kanagawa 252-5210 \\ email: eze@ac.jaxa.jp \\ ${ }^{2}$ Dept. of Astronomy \& Astronomy, University of Nigeria, \\ email: romanus.eze@unn.edu.ng \\ ${ }^{3}$ Dept. of Astronomy, Graduate School of Science, The University of Tokyo, \\ 7-3-1 Hongo, Bunkyo-ku, Tokyo 113-0033
}

\begin{abstract}
The Galactic Ridge X-ray Emission (GRXE) spectrum has strong iron emission lines at $6.4,6.7$, and $7.0 \mathrm{keV}$, each corresponding to the neutral (or low-ionized), He-like, and H-like iron ions. The $6.4 \mathrm{keV}$ fluorescence line is due to irradiation of neutral (or low ionized) material (iron) by hard X-ray sources, indicating uniform presence of the cold matter in the Galactic plane. In order to resolve origin of the cold fluorescent matter, we examined the contribution of the $6.4 \mathrm{keV}$ line emission from white dwarf surfaces in the hard X-ray emitting symbiotic stars (hSSs) and magnetic cataclysmic variables (mCVs) to the GRXE. In our spectral analysis of $4 \mathrm{hSSs}$ and $19 \mathrm{mCVs}$ observed with Suzaku, we were able to resolve the three iron emission lines. We found that the equivalent-widths (EWs) of the $6.4 \mathrm{keV}$ lines of hSSs are systematically higher than those of mCVs, such that the average EWs of hSSs and mCVs are $180_{-10}^{+50} \mathrm{eV}$ and $93_{-3}^{+20} \mathrm{eV}$, respectively. The EW of hSSs compares favorably with the typical EWs of the $6.4 \mathrm{keV}$ line in the GRXE of 90-300 eV depending on Galactic positions. Average $6.4 \mathrm{keV}$ line luminosities of

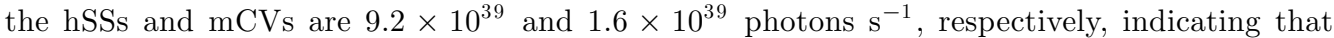
hSSs are intrinsically more efficient $6.4 \mathrm{keV}$ line emitters than mCVs. We estimated required space densities of hSSs and mCVs to account for all the GRXE $6.4 \mathrm{keV}$ line emission flux to be $2 \times 10^{-7} \mathrm{pc}^{-3}$ and $1 \times 10^{-6} \mathrm{pc}^{-3}$, respectively. We also estimated the actual $6.4 \mathrm{keV}$ line contribution from the hSSs, which is as much as $30 \%$ of the observed GRXE flux, and that from the $\mathrm{mCV}$ is about $50 \%$. We therefore conclude that the GRXE $6.4 \mathrm{keV}$ line flux is primarily explained by hSSs and mCVs.
\end{abstract}

Keywords. Galaxy: disk — stars: binaries: symbiotic — stars: novae, cataclysmic variables — X-rays: stars

\section{Introduction}

Presence of the seemingly extended hard X-ray emission from the Galactic Ridge has been known since early 1980's (Galactic Ridge X-ray Emission; GRXE: Worrall et al. (1982), Warwick et al. (1985), Koyama et al. (1986). The nature and origin of this emission is yet to be settled. However, there is a strong argument in favor of collection of faint point sources as opposed to the diffused emission (e.g., Revnivtsev et al. (2006), Krivonos et al. (2007), Revnivtsev et al. (2009), Revnivtsev et al. (2010 and references therein), although the question remains "what are these Galactic point sources?"

We study origin of the $6.4 \mathrm{keV}$ emission line in the GRXE using $4 \mathrm{hSSs}$ and $19 \mathrm{mCVs}$ observed with Suzaku, examining if this emission could be fully resolved by collection of point sources. 

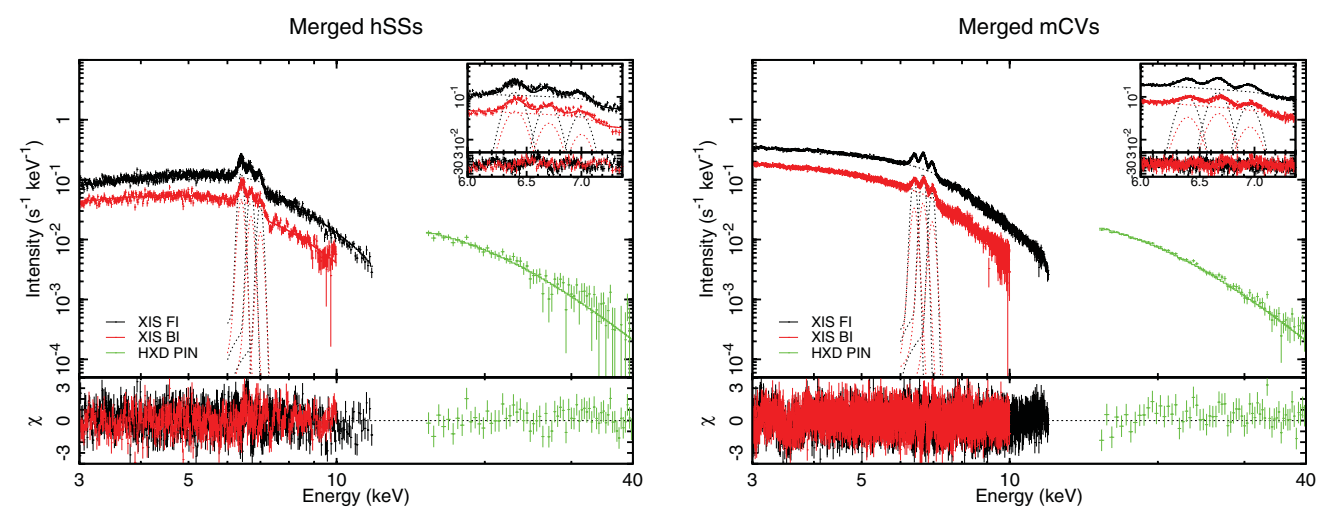

Figure 1. Spectra of the average symbiotic stars(left) and the average magnetic cataclysmic variables(right). In the upper panel, the data and the best-fit model are shown by crosses and solid lines, respectively. In the lower panel, the ratio of the data to the best-fit model is shown by crosses. The inset in the upper panel is an enlarged view for the Fe $\mathrm{K} \alpha$ complex lines.

\section{Spectral Analysis and Results}

Spectral analysis of all observations were performed using XSPEC version 12.7. We modeled the spectrum using absorbed bremsstralung model with three Gaussian lines for the three $\mathrm{Fe} \mathrm{K} \alpha$ emission lines to measure the iron line fluxes. We assumed two types of absorption by full-covering and partial covering matter. The three Fe lines, neutral or low ionized $(6.4 \mathrm{keV})$, He-like $(6.7 \mathrm{keV})$, and $\mathrm{H}$-like $(7.0 \mathrm{keV})$ ions, were clearly resolved in all the sources except in one $\mathrm{mCVs}$, where we were unable to detected the H-like $(7.0 \mathrm{keV})$ significantly but the other two lines were detected.

We used addascaspec to average the spectra of hSSs and mCVs (as well as responses). The spectra for the average hSSs and average $\mathrm{mCVs}$ were presented in figure 1 . We detected strong $6.4 \mathrm{keV}$ iron line emission in the average hSSs spectrum with an equivalent width $(\mathrm{EW})$ of $180_{-10}^{+50} \mathrm{eV}$ and in the average mCVs spectrum with $93_{-3}^{+20} \mathrm{eV}$. We have found that the $6.4 \mathrm{keV}$ line $\mathrm{EW}$ is much stronger in hSSs than in $\mathrm{mCVs}$, which suggests that hSSs can be strong candidates of the GRXE $6.4 \mathrm{keV}$ line emission. For comparison, $6.4 \mathrm{keV}$ iron line EWs of the GRXE are of 90-390 eV, depending on the Galactic locations (Yamauchi et al. 2009).

In our rough estimate, as much as $\sim 30 \%$ of the GRXE $6.4 \mathrm{keV}$ line flux may be from hSSs, and $\sim 50 \%$ from mCVs. We therefore conclude that the GRXE $6.4 \mathrm{keV}$ line flux is primarily explained by hSSs and mCVs.

\section{References}

Koyama, K., Makishima, K., Tanaka, Y., \& Tsunemi, H. 1986, PASJ, 38, 121

Krivonos, R., Revnivtsev, M., Churazov, E., Sazonov, S., Grebenev, S., \& Sunyaev, R. 2007, $A \& A, 463,957$

Revnivtsev, M., Sazonov, S., Gilfanov, M., Churazov, E., \& Sunyaev, R. 2006, Aש̋A, 452, 169

Revnivtsev, M., Sazonov, S., Churazov, E., Froman, W., Vikhlinin, A., \& Sunyaev, R. 2009, Nature, 458, 1142

Revnivtsev, M., van den Berg, M., Burenin, R., Grindlay, J. E., Karasev, D., \& Forman, W. 2010, A\& $A, 515$, A49

Warwick, R. S., Turner, M. J. L., Watson, M. G., \& Willingale, R. 1985, Nature, 317, 218

Worrall, D. M., Marshall, F. E., Boldt, E. A., \& Swank, J. H. 1982, ApJ, 255, 111

Yamauchi, S., et al. 2009, PASJ, 61, S225 